\title{
ON THE AREA OF THE REGION WHERE AN ENTIRE FUNCTION IS GREATER THAN ONE
}

\author{
LI-CHIEN SHEN \\ (Communicated by Paul S. Muhly) \\ To the memory of Professor Robert L. Long
}

ABSTRACT. Using Carleman's inequality, we prove that if $f$ is entire and of finite order $\rho \geq 1$, then

$$
\limsup _{r \rightarrow \infty} \frac{A(r)}{r^{2}} \geq \frac{\pi}{2 \rho}
$$

where $A(r)$ is the area of the region $\{z:|f(z)| \geq 1$ and $|z| \leq r\}$.

1. Introduction. In [2], Edrei and Erdös proved the following

THEOREM A. Let $f$ be an entire function and $D=\{z:|f(z)|>B\}(B>0)$. If there exists a positive number $B$ such that the area of $D$ is finite, then

$$
\liminf _{r \rightarrow \infty} \frac{\ln \ln \ln M(r, f)}{\ln r} \geq 2 \text {. }
$$

In this brief note, we will establish the following

THEOREM 1. Let $f$ be an entire function of order $\rho, 1 \leq \rho<\infty$, and let $A(r)$ denote the area of the region

$$
D_{r}=\{z:|f(z)| \geq 1,|z| \leq r\} .
$$

Then

$$
\limsup _{r \rightarrow \infty} \frac{A(r)}{r^{2}} \geq \frac{\pi}{2 \rho} .
$$

The method, which is different from that of Edrei and Erdös, is based on Carleman's famous inequality which we are about to introduce.

Let $D$ be a region on the complex plane. The boundary of $D$ consists of a finite or infinite number of analytic curves clustering nowhere in the finite complex plane. For any $r, 0<r<\infty$, we denote by $D_{r}$ the part of $D$ lying in $|z|<r$. Let $A_{k}(r)$ $(k=1,2, \ldots, n(r))$ be the arcs of $|z|=r$ contained in $D$ and $r \theta_{k}(r)$ be their arc lengths. Let $E=\{r:|z|=r$ is contained wholly in $D\}$ and $E^{c}=[0, \infty)-E$. If $r \in E^{c}$, we define

$$
\theta(r)=\max _{k} \theta_{k}(r)
$$

For the moment, we leave $\theta(r)$ undefined if $r \in E$.

We now state the following version of Carleman's inequality due to K. Arima [1, p. 64].

Received by the editors March 27, 1986 and, in revised form, October 20, 1986.

1980 Mathematics Subject Classification (1985 Revision). Primary 30D30; Secondary 30D35.

(C) 1988 American Mathematical Society $0002-9939 / 88 \$ 1.00+\$ .25$ per page 
THEOREM B. Let $f$ be an entire function and $D$ be the region where $|f(z)|>1$. Let $\theta(r)$ be defined as before for the region $D$. Then for any $a, 0<a<1$, we have

$$
\ln \ln M(r, f)>\pi \int_{E_{r}^{c}} \frac{d r}{r \theta(r)}-c,
$$

where $E_{r}^{c}=E^{c} \cap[1$, ar $]$ and the constant $c$ depends on a only.

2. New proof of Theorem A. Without loss of generality, we assume $B=1$. We first choose an $a, 0<a<1$ ( $a$ will be fixed throughout $\S \S 2$ and 3). Let $E_{r}=E \cap[1, a r]$, and define $\theta(r)=2 \pi$ for $r \in E$. Then

$$
\pi \int_{E_{r}} \frac{d t}{t \theta(t)}=\frac{1}{2} \int_{E_{r}} \frac{d t}{t} \leq \frac{1}{2} \ln a r .
$$

From (1.2) and (2.1), we have

$$
\pi \int_{1}^{a r} \frac{d t}{t \theta(t)}<\ln \ln M(r, f)+\frac{1}{2} \ln a r+c .
$$

By Schwarz's inequality,

$$
(a r-1)^{2}=\left(\int_{1}^{a r} d t\right)^{2} \leq \int_{1}^{a r} t \theta(t) d t \cdot \int_{1}^{a r} \frac{d t}{t \theta(t)}
$$

We recall that $A(r)=$ area of $\{z:|f(z)| \geq 1$ and $|z| \leq r\}$. Clearly

$$
\int_{1}^{a r} t \theta(t) d t \leq A(a r)
$$

From (2.2), (2.3) and (2.4), we obtain

$$
(a r-1)^{2}<(A(a r) / \pi)\left(\ln \ln M(r, f)+\frac{1}{2} \ln a r+c\right) .
$$

If the area of $D$ is finite, (2.5) clearly implies (1.1). This completes the proof of Theorem A.

3. Proof of Theorem 1. Let

$$
\mu=\liminf _{r \rightarrow \infty} \frac{1}{\ln r} \int_{E_{r}} \frac{d t}{t} \text {. }
$$

Then $0 \leq \mu \leq 1$.

We will prove the following proposition which is slightly more general than Theorem 1.

Proposition 1. Let $f$ be an entire function of order $\rho>0$. Then

$$
\limsup _{r \rightarrow \infty} \frac{A(r)}{r^{2}} \geq \pi \mu+\frac{\pi(1-\mu)^{2}}{2 \rho} \text {. }
$$

Proof. From Schwarz's inequality,

$$
\left(\int_{E_{r}^{c}} \frac{1}{t} d t\right)^{2} \leq \int_{E_{r}^{c}} \frac{\theta(t)}{t} d t \cdot \int_{E_{r}^{c}} \frac{1}{t \theta(t)} d t .
$$

Combining (3.1) with Carleman's inequality, we obtain

$$
\int_{E_{r}^{c}} \frac{\theta(t)}{t} d t \geq \pi\left(\int_{E_{r}^{c}} \frac{d t}{t}\right)^{2} /(\ln \ln M(r, f)+c) .
$$


We again define $\theta(r)=2 \pi$ for $r \in E$. Then

$$
\begin{aligned}
\int_{E_{r}^{c}} \frac{\theta(t)}{t} d t & =\int_{1}^{a r} \frac{\theta(t)}{t} d t-\int_{E_{r}} \frac{\theta(t)}{t} d t \\
& =\int_{1}^{a r} \frac{\theta(t)}{t} d t-2 \pi \int_{E_{r}} \frac{d t}{t} .
\end{aligned}
$$

Let $B(r)=\int_{1}^{r} t \theta(t) d t$. Clearly, $B(r) \leq A(r)$ for all $r \geq 1$. We therefore have

$$
\begin{aligned}
\int_{1}^{a r} \frac{\theta(t)}{t} d t & =\int_{1}^{a r} \frac{d B(t)}{t^{2}}=2 \int_{1}^{a r} \frac{B(t)}{t^{3}} d t+K(r) \\
& \leq 2 \int_{1}^{a r} \frac{A(t)}{t^{3}} d t+K(r),
\end{aligned}
$$

where $K(r)=\left(B(a r) / a^{2} r^{2}-B(1)\right)$. We note that $B(r) / r^{2} \leq \pi$ for all $r$. From (3.2), (3.3) and (3.4), we conclude that

$$
\frac{K(r)}{\ln r}+\frac{2}{\ln r} \int_{1}^{a r} \frac{A(t)}{t^{3}} d t \geq \frac{2 \pi}{\ln r} \int_{E_{r}} \frac{d t}{t}+\pi\left(\frac{1}{\ln r} \int_{E_{r}^{c}} \frac{d t}{t}\right)^{2} /\left(\frac{\ln \ln M(r, f)+c}{\ln r}\right) .
$$

It follows immediately from (3.5) that

$$
\limsup _{r \rightarrow \infty} \frac{A(r)}{r^{2}} \geq \pi \mu+\frac{\pi(1-\mu)^{2}}{2 \rho} .
$$

This finishes the proof of Proposition 1 .

It is easy to verify that, if $\rho \geq 1$.

$$
\pi \mu+\frac{\pi(1-\mu)^{2}}{2 \rho} \geq \frac{\pi}{2 \rho}
$$

Theorem 1 follows from (3.6).

REMARK. From (3.5), we see that if $\rho=0$, then $\mu=1$. This yields

$$
\limsup _{r \rightarrow \infty} \frac{A(r)}{r^{2}}=\pi
$$

We also note that Proposition 1 gives $\mu+(1-\mu)^{2} / 2 \rho \leq 1$ for $0<\rho<1$. This provides the following relation for $\mu$ and $\rho$ :

$$
1-\frac{\rho}{2} \leq \mu \leq 1
$$

We also point out here that the conclusion of Theorem 1 is sharp, as may be seen by considering Mittag-Leffler's function $E_{1 / \rho}$.

\section{REFERENCES}

1. K. Arima, On maximum modulus of integral functions, J. Math. Soc. Japan 4 (1952), 62-66.

2. A. Edrei and P. Erdös, Entire functions bounded outside a finite area, Acta Math. Hungar. 45 (1985), 367-376.

Department of Mathematics, University of Florida, Gainesville, Florida 32611 